\title{
Constraining the rate of GRB visible afterglows with the CFHTLS very wide survey ${ }^{\star}$
}

\author{
F. Malacrino ${ }^{1}$, J.-L. Atteia ${ }^{1}$, M. Boër ${ }^{2}$, A. Klotz ${ }^{2,3}$, C. Veillet ${ }^{4}$, J.-C. Cuillandre ${ }^{4}$, and the GRB RTAS collaboration ${ }^{\star \star}$ \\ ${ }^{1}$ Laboratoire d'Astrophysique de Toulouse-Tarbes, Observatoire Midi-Pyrénées (CNRS-UMR5572/Université Paul Sabatier \\ Toulouse III), 14 avenue Édouard Belin, 31400 Toulouse, France \\ e-mail: fmalacri@ast.obs-mip.fr \\ 2 Observatoire de Haute-Provence, 04870 Saint-Michel l'Observatoire, France \\ 3 Centre d'Étude Spatiale des Rayonnements, Observatoire Midi-Pyrénées (CNRS/UPS), BP 4346, 31028 Toulouse Cedex 04, France \\ ${ }^{4}$ Canada-France-Hawaii Telescope Corp., Kamuela, HI 96743, USA
}

Received 11 December 2006 / Accepted 19 January 2007

\section{ABSTRACT}

\begin{abstract}
Aims. We analyze images of the CFHTLS Very Wide Survey to search for visible orphan afterglows from gamma-ray bursts (GRBs). Methods. We have searched 490 square degrees down to magnitude $r^{\prime}=22.5$ for visible transients similar to GRB afterglows. We translate our observations into constraints on the number of GRB visible afterglows in the sky, by measuring the detection efficiency of our search with a simulation reproducing the characteristics of our observational strategy and the properties of on-axis GRB afterglows.

Results. We have found only three potential candidates, of which two are most probably variable stars, and one presents similarities to an orphan afterglow. We constrain the number of visible afterglows to be less than 220 down to $r^{\prime}=22.5$ in the whole sky at any time. Our observations are marginally consistent with the most optimistic model, which predicts orphan afterglows to be about 10 times more frequent than GRBs.

Conclusions. This search has led to the detection of one possible GRB afterglow, and provides the strongest constraints on the rate of GRB visible afterglows as well as an estimation of the observing time required to detect a significant number of GRB afterglows.
\end{abstract}

Key words. gamma rays: bursts - methods: data analysis

\section{Introduction}

The prediction of the existence of orphan GRB afterglows relies on the double assumption that the GRB prompt emission is beamed and that the afterglow emission is still bright enough to be detectable when it starts to radiate outside the GRB beam. This situation, which was described by Rhoads in 1997 soon after the discovery of the first GRB afterglows, makes the detection of visible afterglows possible even without the GRB trigger. Such afterglows are usually called "orphan afterglows". The properties and expected number of orphan GRB afterglows have been discussed by Rhoads (1997, 1999), Totani \& Panaitescu (2002), Nakar et al. (2002), and Dalal et al. (2002). Huang et al. (2002) have proposed that failed on-axis GRBs with Lorentz factors well below 100 could also produce orphan afterglows.

The detection of orphan afterglows at optical wavelengths would open a new window in the GRB field. First, it would

\footnotetext{
* Based on observations obtained with MegaPrime/MegaCam, a joint project of CFHT and CEA/DAPNIA, at the Canada-France-Hawaii Telescope (CFHT) which is operated by the National Research Council (NRC) of Canada, the Institut National des Sciences de l'Univers of the Centre National de la Recherche Scientifique (CNRS) of France, and the University of Hawaii.

$\star \star$ Collaboration of researchers who proposed the GRB RTAS project (see http://www. cfht.hawaii. edu/ grb/).
}

suppress the existing bias in GRB studies due to the fact that all GRBs observed to date have been detected by their prompt emission at high-energies. Second, since orphan afterglows are thought to be far more numerous than on-axis afterglows, we expect to detect many more nearby afterglows, allowing detailed studies on specific issues like the GRB-SN connection. Third, the detection, or non-detection, of orphan afterglows will provide constraints on the beaming angle and energetics of GRBs (see Rhoads 1997; Totani \& Panaitescu 2002; Nakar et al. 2002).

Given the potential science returns from the detection of visible orphan afterglows, various searches have been performed, with very different depth and sky coverage (Becker et al. 2004; Rykoff et al. 2005; Rau et al. 2006). These searches produced no orphan afterglow detection and provided constraints on GRB beaming which are described in Sect. 3. We report here the results of a search performed in images taken for the CFHTLS Very Wide Survey (hereafter CFHTLS-VWS) which has a combination of depth and sky coverage providing an unprecedented sensitivity for orphan afterglow searches. An extensive description of the survey and of our search procedure has been given in Malacrino et al. (2006, hereafter Paper I), we refer the reader to this paper for details. In this letter we focus on the afterglow candidates found in the CFHTLS-VWS images (Sect. 2) and on the constraints that we derive on the frequency of GRB visible afterglows (Sect. 3). 
Table 1. Summary of our observations. The columns give for each filter: the number of images processed, $N_{\text {im }}$, the surface area in square degree, $S_{\text {im }}$, the mean accuracy of the astrometry in arcsecond, the completeness magnitude, $M_{\text {lim }}$, the density of astronomical sources per square degree, and the percentage of images that have been properly processed (see Paper I).

\begin{tabular}{ccccccc}
\hline \hline Filter & $N_{\mathrm{im}}$ & $\begin{array}{c}S_{\mathrm{im}} \\
{\left[\mathrm{deg}^{2}\right]}\end{array}$ & $\begin{array}{c}\text { Accuracy } \\
\operatorname{arcsec}\end{array}$ & $M_{\lim }$ & $\begin{array}{c}\text { Density } \\
\mathrm{deg}^{-2}\end{array}$ & Success \\
\hline$g^{\prime}$ & 536 & 481 & 0.52 & 23.1 & 31910 & 99.47 \\
$r^{\prime}$ & 1302 & 1167 & 0.54 & 22.6 & 41370 & 99.30 \\
$i^{\prime}$ & 589 & 531 & 0.51 & 22.4 & 51075 & 99.83 \\
\hline
\end{tabular}

\section{Visible afterglow candidates}

The present work is based on the analysis of images covering an area of 490 square degrees. Their completeness magnitude depends on the filter. $50 \%$ of our images are complete down to $g^{\prime}=23.1, r^{\prime}=22.6$, and $i^{\prime}=22.4$ (see Table 1). The regions of the sky that have been observed, and the analysis of the images leading to the detection and validation of afterglow candidates are described in detail in Paper I. We briefly recall here only the points relevant to our orphan afterglow search.

When a new image is recorded for the CFHTLS-VWS, we construct the catalog of sources in the image. Usually, three images of the same field are acquired about $1 \mathrm{~h}$ apart during a single night, and another one on the following night. Our software compares the sources detected in these images and searches for photometrically variable or moving objects, which are checked by a member of the collaboration less than $24 \mathrm{~h}$ after the acquisition of the images (see Paper I for details). A visual examination of these objects led to the rejection of $90 \%$ of them, and to the validation of the remaining $10 \%$ as truly variable objects, which correponds to $0.007 \%$ of the total number of sources.

While Paper I discusses variable sources found in all comparisons, we restrict the work in this paper to sources found in inter-night comparisons. This is not a strong limitation since all afterglows should be detected in inter-night comparisons (see Paper I). Another important point is that we have chosen to restrict our search to objects that vary by 0.2 mag or more, a value that offers a good compromise between sensitivity and the number of false detections. Our analysis has led to the identification of 1067 truly variable objects out of more than 18 million, in 549 MegaCAM fields, which shows that few objects in the sky show variations larger than $0.2 \mathrm{mag}$ on a timescale of 1 day. The absolute variation in magnitude of these sources between the two nights as a function of their median magnitude is shown in Fig. 1.

All the objects of Fig. 1 have been carefully examined in order to determine their nature. Most of them are variable stars which are identified as such because they are present in archived astronomical images ${ }^{1}$, or in the USNO-B1.0 catalog (Monet et al. 2003), or in images of the same field acquired during other observational periods of the CFHTLS-VWS. This last method is particularly useful for variable objects fainter than the limit of the USNO-B1.0 catalog (about 21st mag). This selection assumes that GRB afterglows are truly transient sources. During this screening process we also eliminate a few slow moving objects that are referenced in the MPchecker (http://scully.harvard.edu/ cgi/CheckMP). These objects can mimick a GRB afterglow that has "disappeared" after

\footnotetext{
${ }^{1}$ We use the Aladin sky atlas, Bonnarel et al. (2000).
}

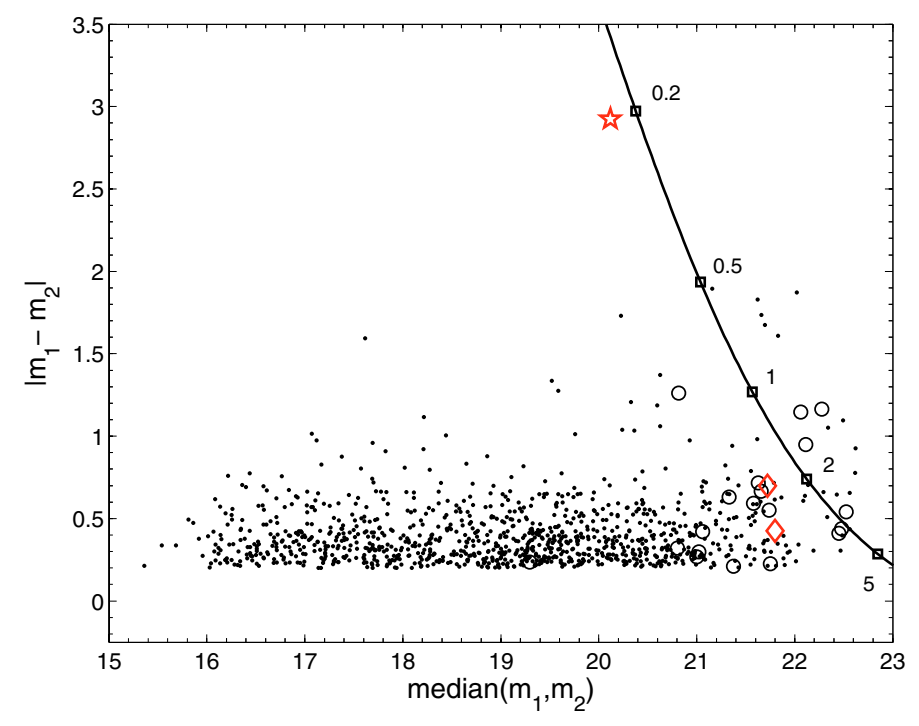

Fig. 1. 1067 variable objects detected by our automatic software and confirmed by visual examination. Each point represents a single object in the inter-night comparison of a pair of images. The $x$-axis shows the median magnitude while the $y$-axis shows the absolute value of the magnitude difference between the two images. The open circles and the two red diamonds show the objects that have been identified as afterglow candidates in the real-time analysis, and discarded (circles), or not (diamonds) after further analysis (see text). The solid line shows where a typical afterglow $\left(\alpha=1.2, m_{1}=21\right.$ and $\left.m_{\text {host }}=24\right)$ would be placed on this diagram as a function of its age (in days) at the time of the first observation. The red star shows OT 20050728, a variable source which has characteristics similar to GRB afterglows.

one day. Variable sources that are not eliminated by one of these methods are classified as "afterglow candidates".

During the real-time process, 26 sources were classified as "afterglow candidates". A careful re-analysis of these sources with the full set of images allowed us to re-qualify 23 of them as variable stars. These 23 sources are shown with open circles in Fig. 1. We note that nearly all of them are fainter than the magnitude limit of USNO-B1.0, emphasizing the role of this catalog in the rejection of bright variable sources. At the time of writing this paper we are left with only 3 objects, listed in Table 2 . Two of them (the diamonds) are probably variable stars which we still consider as "candidates" due to the lack of reference images. The last transient (the red $\star$ in Fig. 1) is a remarkable event which shares some similarities with a visible afterglow (the solid line in Fig. 1 simulates the track of a typical on-axis afterglow). We have called it OT 20050728, and its evolution is shown in Fig. 2. Unfortunately, the available data (see Table 2) are not sufficient to unambiguously determine its nature.

One significant source of background in searches for GRB visible afterglows is flare stars (Kulkarni \& Rau 2006). We consider that it is unlikely that OT 20050728 is a stellar flare since its long rise time (greater than $1 \mathrm{~h}$ ) and its 3 mag variation in the $i^{\prime}$ band are unusual for stellar flares. We thus consider OT 20050728 as a possible visible afterglow candidate. In view of the importance of the characterization of this event, we have requested additional observations during June and July 2007. We strongly encourage deep observations of this source.

In the following we discuss the rate of GRB visible afterglows under two assumptions; i) our search has led to the detection of zero afterglow; ii) OT 20050728 is a GRB afterglow. 


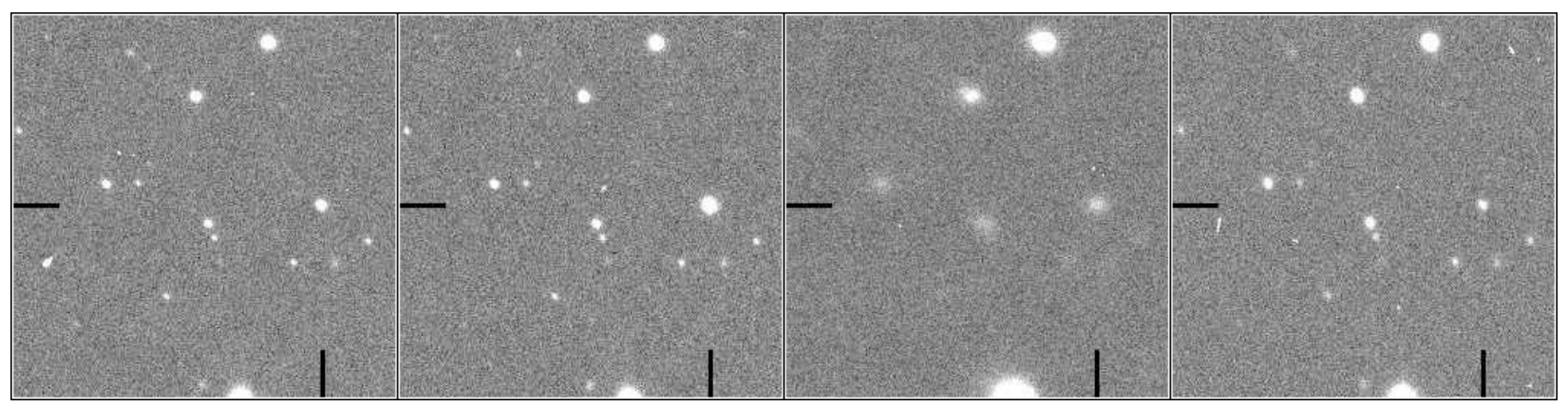

Fig. 2. The evolution of OT 20050728 during the nights of 2005 July 28th and 29th. The 4 images correspond to the 4 lines in bold in Table 2.

Table 2. Properties of our afterglow candidates: name, Right Ascension, Declination, number of images available, date and time of the observations, magnitude of the source, and a comment on the most probable nature of the source.

\begin{tabular}{lllllllll}
\hline \hline Name & RA & Dec & $N_{\text {im }}$ & Date & Time [SOD] & Filter & Magnitude & Comment \\
\hline OT 20050629 & 193806.81 & -212231.3 & 4 & $2005-06-29$ & 38182 & $r^{\prime}$ & 21.54 & probably a variable star, \\
& & & & $2005-06-29$ & 40527 & $r^{\prime}$ & 21.55 & only 4 images \\
& & & & $2005-06-29$ & 42924 & $r^{\prime}$ & 21.37 & \\
\hline OT 20050728 & 155716.78 & -185058.6 & 11 & $2005-07-01$ & 42422 & $r^{\prime}$ & 22.07 & \\
& & & & $2005-06-10$ & 34244 & $g^{\prime}$ & $>23.35$ & afterglow candidate, \\
& & & & 22204 & $g^{\prime}$ & $>23.19$ & detected in 5 out of 11 images \\
& & & & $\mathbf{2 0 0 5 - 0 7 - 2 8}$ & $\mathbf{2 1 8 4 9}$ & $i^{\prime}$ & $\mathbf{2 0 . 0 8}$ & \\
& & & & $\mathbf{2 0 0 5 - 0 7 - 2 8}$ & $\mathbf{2 6 0 5 4}$ & $i^{\prime}$ & $\mathbf{1 8 . 6 6}$ & \\
& & & & $2005-07-28$ & 30330 & $i^{\prime}$ & 20.90 & \\
\hline OT 20060202 & 045405.71 & \multirow{2}{*}{+214517.8} & 4 & $2006-02-02$ & 26962 & $i^{\prime}$ & 21.58 & probably a variable star, \\
& & & & $2006-02-02$ & 30226 & $i^{\prime}$ & 21.74 & only 4 images \\
& & & & $2006-02-02$ & 33356 & $i^{\prime}$ & 21.91 & \\
\end{tabular}

\section{Constraining GRB beaming}

In this section we use our observations to constrain the number of visible afterglows in the whole sky. The transformation of this value into a number of detected afterglows in a given survey depends on parameters describing the observational strategy (sky coverage, limiting magnitude, time between consecutive observations of a given field...) and on parameters describing the afterglows (shape of the light-curve, magnitude at 1 day, magnitude of the host...). We have constructed a simple simulation which generates random afterglow light-curves and computes the fraction detected in a given observational strategy (see Paper I for more details). One current limitation of our simulation is that it uses observed light-curves taken from the GCN Circulars, which are presumably generated by on-axis GRBs. This may not be too much of a problem however because in a deep survey like ours, GRB afterglows are detected after several days, when the lightcurves of on-axis and off-axis afterglows are similar. According to our simulation, and assuming that we have detected no afterglow, the number of visible afterglows in the sky at a given time down to $r^{\prime}=22.5$ is smaller than 220 (90\% confidence). This translates into an upper limit of 10100 visible afterglows per year, or less than 13 orphan afterglows per GRB. If we assume in contrast that we have detected one afterglow, the number of afterglows in the sky at a given time is 100, with an error bar comprised between 10 and 350 (90\% confidence). These numbers are reported in Fig. 3 along with the constraints derived from previous searches and some theoretical estimates.

Figure 3 shows that our search is about 10 times more sensitive than previous works. This is due to its unprecedented combination of depth and sky coverage which has been permitted by

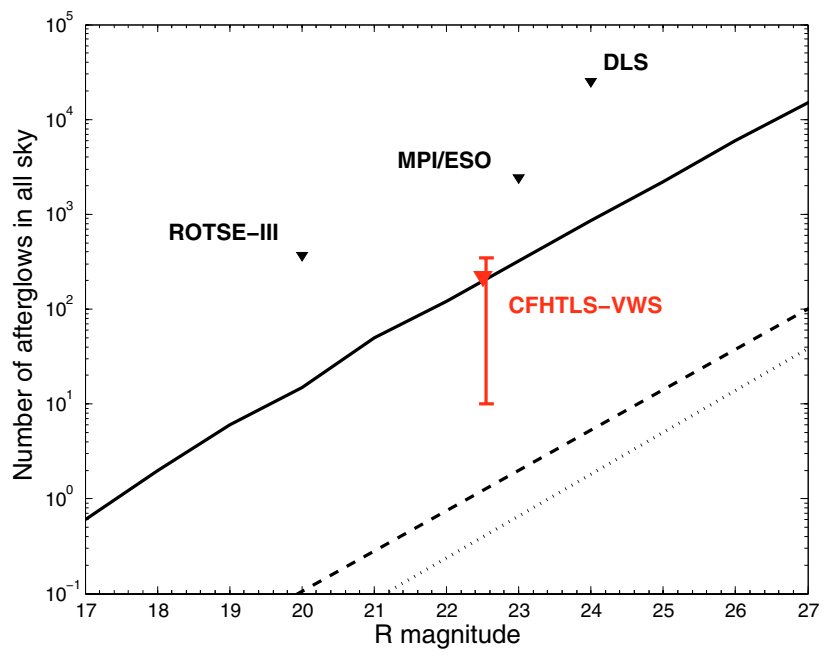

Fig. 3. Constraints provided by our observations (VWS), compared to the results of ROTSE-III (Rykoff et al. 2005), of the MPI/ESO survey (Rau et al. 2006), and of the DLS (Becker et al. 2004). The upper limit assumes the detection of zero afterglow and the error bar the detection of one afterglow in our survey (see Sect. 2 for additional explanations). Also shown are the theoretical predictions of Totani \& Panaitescu (2002, solid line), of Nakar et al. (2002, dashed line), and of Zou et al. (2007, dotted line).

the large area of MegaCAM, the high throughput of the $3.6 \mathrm{~m}$ $\mathrm{CFH}$ Telescope, the organisation of a survey adequate for visible afterglow searches, and by the implementation of dedicated software allowing a quick processing of the images and 
an efficient search for variable objects. If we compare our observations to theoretical predictions, the assumption that we have detected no afterglow gives an upper limit which is marginally consistent with the (optimistic) prediction of Totani \& Panaitescu (2002), and fully consistent with the more pessimistic predictions of Nakar et al. (2002), and Zou et al. (2007). If we assume that our candidate is a real afterglow we reach a different conclusion: our data are incompatible with the predictions of Nakar et al. (2002) and Zou et al. (2007) at the 90\% confidence level. More importantly, if OT 20050728 is a true GRB afterglow, our work is the first one to provide an estimate of the amount of observing time needed to detect a significant number of GRB visible afterglows.

While the detection of orphan afterglows was initially proposed as a way to constrain GRB beaming (Rhoads 1997), more recent work has shown that the number of visible afterglows depends on various factors like the structure of the jet (Dalal et al. 2002; Granot et al. 2002; Nakar et al. 2002; Totani \& Panaitescu 2002) and on the existence of orphan afterglows not due to off-axis GRBs (Huang et al. 2002; Nakar \& Piran 2003). It could be possible to constrain the GRB beaming by including a model of GRB jets in our simulations, but this is beyond the scope of this paper.

Along the duration of the survey our main method to discard false alarms in a search for GRB afterglows has been the comparison with images taken months or years earlier or later. In doing so we rely on the truly transient nature of GRB afterglows, which is one of their most unique properties. This is illustrated in Fig. 1, which shows that, among 22 million objects, we have found one thousand sources varying by more than 0.2 mag in one day, and that only one of these sources was truly transient. We believe that future efficient searches should be made in regions of the sky covering several tens to a few hundred square degrees, already observed down to $m \sim 24-25$, and which are outside the Ecliptic and Galactic planes. This is typically the case of weak shear surveys, like the CFHTLS Wide Synoptic Survey which covers a total of 175 square degrees in 5 filters $\left(u^{*}, g^{\prime}, r^{\prime}, i^{\prime}, z^{\prime}\right)$, down to $i^{\prime}=24.5$. The existence of such catalogs represents a remarkable opportunity for future searches of GRB visible afterglows. Moreover, the combination of optical and radio observations (Levinson et al. 2002; Gal-Yam et al. 2006) may be the best way to measure GRB beaming in the near future.

Acknowledgements. We thank everyone at CFHT for their continuous support, especially Kanoa Withington. We thank the Observatoire Midi-Pyrénées for funding the Real Time Analysis System.

\section{References}

Becker, A. C., Wittman, D. M., Boeshaar, P. C., et al. 2004, ApJ, 611, 418 Bonnarel, F., Fernique, P., Bienayme, O., et al. 2000, A\&AS, 143, 33 Dalal, N., Griest, K., \& Pruet, J. 2002, ApJ, 564, 209

Gal-Yam, A., Ofek, E. O., Poznanski, D., et al. 2006, ApJ, 639, 331 Granot, J., Panaitescu, A., Kumar, P., \& Woosley, S. E. 2002, ApJ, 570, L61 Huang, Y. F., Dai, Z. G., \& Lu, T. 2002, MNRAS, 332, 735 Kulkarni, S. R., \& Rau, A. 2006, ApJ, 644, L63

Levinson, A., Ofek, E. O., Waxman, E., \& Gal-Yam, A. 2002, ApJ, 576, 923

Malacrino, F., Atteia, J-L., Boër, M., et al. 2006, A\&A, 459, 465 (Paper I)

Monet, D. G., Levine, S. E., Canzian, B., et al. 2003, AJ, 125, 984

Nakar, A., \& Piran, T. 2003, NewA, 8, 141

Nakar, A., Piran, T., \& Granot, J. 2002, ApJ, 579, 699

Rau, A., Greiner, J., \& Schwarz, R. 2006, A\&A, 449, 79

Rhoads, J. E. 1997, ApJ, 487, L1

Rhoads, J. E. 1999, ApJ, 525, 737

Rykoff, E. S., Aharonian, F., Akerlof, C. W., et al. 2005, ApJ, 631, 1032

Totani, T., \& Panaitescu, A. 2002, ApJ, 576, 120

Zou, Y. C., Wu, X. F., \& Dai, Z. G. 2007, A\&A, 461, 115 